Supporting Information Belonging to the Publication

\title{
Benzimidazolin-2-stannylenes with N,N'-Alkyl (Me and Et) and Lewis Base Functional Groups
}

\author{
F. Ekkehardt Hahn, ${ }^{*}{ }^{\dagger}$ Lars Wittenbecher, ${ }^{\dagger}$ D. Le Van, ${ }^{\dagger}$ Alexander V. Zabula ${ }^{\dagger, \ddagger}$ \\ Institut für Anorganische und Analytische Chemie der Westfälischen Wilhelms-Universität Münster and \\ NRW Graduate School of Chemistry, Corrensstrasse 36, D-48149 Münster, Germany
}

Spectroscopic data for the compounds $1 \mathrm{a}-1 \mathrm{1o}, 2 \mathrm{a}-2 \mathrm{~d}, 2 \mathrm{~g}-2 \mathrm{i}$, and $2 \mathrm{~m}-2 \mathrm{2o}$.

$\boldsymbol{N}, \boldsymbol{N}^{\prime}$-Bis(formyl)-1,2-diamidobenzene (1a). Colourless solid. Yield: $91 \%$. ${ }^{1} \mathrm{H}$ NMR $\left(250 \mathrm{MHz}, d_{6^{-}}\right.$ DMSO): $\delta 9.55(\mathrm{~m}, 2 \mathrm{H}, \mathrm{NHC}(\mathrm{O})), 8.20(\mathrm{~m}, 2 \mathrm{H}, H \mathrm{C}(\mathrm{O})), 7.62(\mathrm{~m}, 2 \mathrm{H}, \mathrm{Ar}-\mathrm{H}), 7.14(\mathrm{~m}, 2 \mathrm{H}, \mathrm{Ar}-\mathrm{H})$. ${ }^{13} \mathrm{C}$ NMR (62.9 MHz, $d_{6}$-DMSO): $\delta 160.3(\mathrm{NHC}(\mathrm{O})), 129.4\left(\mathrm{Ar}^{-} \mathrm{C}_{\text {ipso }}\right), 125.2\left(\mathrm{Ar}-\mathrm{C}_{\text {meta }}\right), 124.2$ $\left(\mathrm{Ar}-\mathrm{C}_{\text {ortho }}\right)$

$N, N^{\prime}$-Bis(acetyl)-1,2-diamidobenzene (1b). Colourless solid. Yield: $96 \% .{ }^{1} \mathrm{H}$ NMR $\left(250 \mathrm{MHz}, d_{6}\right.$ DMSO): $\delta 9.41(\mathrm{~s}, 2 \mathrm{H}, \mathrm{NH}), 7.53(\mathrm{~m}, 2 \mathrm{H}, \mathrm{Ar}-\mathrm{H}), 7.12(\mathrm{~m}, 2 \mathrm{H}, \mathrm{Ar}-\mathrm{H}), 2.02\left(\mathrm{~s}, 6 \mathrm{H}, \mathrm{CH}_{3}\right) .{ }^{13} \mathrm{C} \mathrm{NMR}$ (62.9 MHz, $d_{6}$-DMSO): $\delta 168.6(\mathrm{NHC}(\mathrm{O})), 130.5\left(\mathrm{Ar}-\mathrm{C}_{i p s o}\right), 124.5\left(\mathrm{Ar}-\mathrm{C}_{\text {meta }}\right), 123.6\left(\mathrm{Ar}-\mathrm{C}_{\text {ortho }}\right), 23.7$ $\left(\mathrm{CH}_{3}\right)$. 
$\boldsymbol{N}, \boldsymbol{N}^{\prime}$-Bis(2-methoxy-acetyl)-1,2-diamidobenzene (1c). Brownish solid. Yield: $89 \%$. ${ }^{1} \mathrm{H}$ NMR (250 $\left.\mathrm{MHz}, \mathrm{CD}_{3} \mathrm{CN}\right): \delta 8.80(\mathrm{~s}, 2 \mathrm{H}, \mathrm{NHC}(\mathrm{O})), 7.55(\mathrm{~m}, 2 \mathrm{H}, \mathrm{Ar}-\mathrm{H}), 7.20(\mathrm{~m}, 2 \mathrm{H}, \mathrm{Ar}-\mathrm{H}), 4.00\left(\mathrm{~s}, 4 \mathrm{H}, \mathrm{CH}_{2}\right)$, $3.45\left(\mathrm{~s}, 6 \mathrm{H}, \mathrm{CH}_{3}\right) .{ }^{13} \mathrm{C} \mathrm{NMR}\left(62.9 \mathrm{MHz}, \mathrm{CD}_{3} \mathrm{CN}\right): \delta 169.9(\mathrm{NHC}(\mathrm{O})), 131.2\left(\mathrm{Ar}-\mathrm{C}_{\text {ipso }}\right), 126.9$ $\left(\mathrm{Ar}-\mathrm{C}_{\text {meta }}\right), 126.1\left(\mathrm{Ar}-\mathrm{C}_{\text {ortho }}\right), 72.7\left(\mathrm{CH}_{2}\right), 59.8\left(\mathrm{CH}_{3}\right)$.

$\boldsymbol{N}, \boldsymbol{N}^{\prime}$-Bis(3-methoxy-propionyl)-1,2-diamidobenzene (1d). Brown solid. Yield: $90 \%$. ${ }^{1} \mathrm{H}$ NMR (250 $\left.\mathrm{MHz}, \mathrm{CDCl}_{3}\right): \delta 8.53(\mathrm{~s}, 2 \mathrm{H}, \mathrm{NHC}(\mathrm{O})), 7.51(\mathrm{~m}, 2 \mathrm{H}, \mathrm{Ar}-\mathrm{H}), 7.13(\mathrm{~m}, 2 \mathrm{H}, \mathrm{Ar}-\mathrm{H}), 3.71\left(\mathrm{t}, 2 \mathrm{H}, \mathrm{CH}_{2} \mathrm{O}\right)$, $3.40\left(\mathrm{~s}, 6 \mathrm{H}, \mathrm{CH}_{3}\right), 2.68\left(\mathrm{t}, 2 \mathrm{H}, \mathrm{CH}_{2} \mathrm{C}(\mathrm{O})\right) .{ }^{13} \mathrm{C} \mathrm{NMR}\left(62.9 \mathrm{MHz}, \mathrm{CDCl}_{3}\right): \delta 170.5(\mathrm{NHC}(\mathrm{O})), 130.3$ $\left(\mathrm{Ar}-\mathrm{C}_{i p s o}\right), 126.1\left(\mathrm{Ar}-\mathrm{C}_{\text {meta }}\right), 125.3\left(\mathrm{Ar}-\mathrm{C}_{\text {ortho }}\right), 68.4\left(\mathrm{CH}_{2} \mathrm{O}\right), 58.9\left(\mathrm{CH}_{2} \mathrm{C}(\mathrm{O})\right), 37.1\left(\mathrm{CH}_{3}\right)$.

$\boldsymbol{N}, \boldsymbol{N}^{\prime}$-Bis(2-chloro-acetyl)-1,2-diamidobenzene (1e). Light red solid. Yield: $90 \%$. ${ }^{1} \mathrm{H}$ NMR $(250 \mathrm{MHz}$ $d_{6}$-DMSO): $\delta 9.61(\mathrm{~s}, 2 \mathrm{H}, \mathrm{NHC}(\mathrm{O})), 7.50(\mathrm{~m}, 2 \mathrm{H}, \mathrm{Ar}-\mathrm{H}), 7.18(\mathrm{~m}, 2 \mathrm{H}, \mathrm{Ar}-\mathrm{H}), 4.42\left(\mathrm{~s}, 4 \mathrm{H}, \mathrm{CH}_{2} \mathrm{C}(\mathrm{O})\right)$. ${ }^{13} \mathrm{C}$ NMR (62.9 MHz, $d_{6}$-DMSO): $\delta 165.2(\mathrm{NHC}(\mathrm{O})), 130.2\left(\mathrm{Ar}-\mathrm{C}_{\text {ipso }}\right), 125.7\left(\mathrm{Ar}-\mathrm{C}_{\text {meta }}\right), 125.1$ $\left(\mathrm{Ar}-\mathrm{C}_{\text {ortho }}\right), 43.3\left(\mathrm{CH}_{2} \mathrm{C}(\mathrm{O})\right)$.

$\boldsymbol{N}, \boldsymbol{N}^{\prime}$-Bis(3-chloro-propionyl)-1,2-diamidobenzene (1f). Brownish solid. Yield: $82 \%$. ${ }^{1} \mathrm{H}$ NMR (250 $\mathrm{MHz}, d_{6}$-DMSO): $\delta 9.45(\mathrm{~s}, 2 \mathrm{H}, \mathrm{NHC}(\mathrm{O})), 7.55(\mathrm{~m}, 2 \mathrm{H}, \mathrm{Ar}-\mathrm{H}), 7.15(\mathrm{~m}, 2 \mathrm{H}, \mathrm{Ar}-\mathrm{H}), 3.90(\mathrm{t}, 2 \mathrm{H}$, $\left.\mathrm{CH}_{2} \mathrm{C}(\mathrm{O})\right), 2.80\left(\mathrm{t}, 2 \mathrm{H}, \mathrm{CH}_{2} \mathrm{Cl}\right) .{ }^{13} \mathrm{C} \mathrm{NMR}\left(62.9 \mathrm{MHz}, d_{6}\right.$-DMSO): $\delta 168.9(\mathrm{NHC}(\mathrm{O})), 130.8\left(\mathrm{Ar}-\mathrm{C}_{i p s o}\right)$, 125.6 (Ar- $\left.\mathrm{C}_{\text {meta }}\right), 125.4\left(\mathrm{Ar}-\mathrm{C}_{\text {ortho }}\right), 41.3\left(\mathrm{CH}_{2} \mathrm{C}(\mathrm{O})\right), 39.0\left(\mathrm{CH}_{2} \mathrm{Cl}\right)$.

$\boldsymbol{N}, \boldsymbol{N}^{\prime}$-Bis(2-dimethylamino-acetyl)-1,2-diamidobenzene (1g). Brownish solid. Yield: 82\%. ${ }^{1} \mathrm{H}$ NMR (250 MHz, $d_{6}$-DMSO): $\delta 9.2$ (brs, 2H, NHC(O)), $7.43(\mathrm{~m}, 2 \mathrm{H}, \mathrm{Ar}-\mathrm{H}), 6.71(\mathrm{~m}, 2 \mathrm{H}, \mathrm{Ar}-\mathrm{H}), 2.96(\mathrm{~s}, 4 \mathrm{H}$, $\left.\mathrm{CH}_{2} \mathrm{C}(\mathrm{O})\right), 2.06\left(\mathrm{~s}, 6 \mathrm{H}, \mathrm{CH}_{3}\right) .{ }^{13} \mathrm{C} \mathrm{NMR}\left(62.9 \mathrm{MHz}, d_{6}\right.$-DMSO): $\delta 168.6(\mathrm{NHC}(\mathrm{O})), 29.2\left(\mathrm{Ar}-\mathrm{C}_{i p s o}\right)$, 125.2( $\left(\mathrm{Ar}-\mathrm{C}_{\text {meta }}\right), 123.9\left(\mathrm{Ar}-\mathrm{C}_{\text {ortho }}\right), 62.2\left(\mathrm{CH}_{2} \mathrm{C}(\mathrm{O})\right), 45.1\left(\mathrm{CH}_{3}\right)$. 
$\boldsymbol{N}, \boldsymbol{N}^{\prime}$-Bis(3-dimethylamino-propionyl)-1,2-diamidobenzene (1h). Brownish solid. Yield: $81 \%$. ${ }^{1} \mathrm{H}$ NMR (250 MHz, $d_{6}$-DMSO): $\delta 10.05(\mathrm{~s}, 2 \mathrm{H}, \mathrm{NHC}(\mathrm{O})), 7.55(\mathrm{~m}, 2 \mathrm{H}, \mathrm{Ar}-\mathrm{H}), 7.10(\mathrm{~m}, 2 \mathrm{H}, \mathrm{Ar}-\mathrm{H}), 3.15$ $\left(\mathrm{t}, 4 \mathrm{H}, \mathrm{CH}_{2} \mathrm{C}(\mathrm{O})\right), 2.80\left(\mathrm{t}, 2 \mathrm{H}, \mathrm{CH}_{2} \mathrm{~N}\right), 2.55\left(\mathrm{~s}, 12 \mathrm{H}, \mathrm{CH}_{3}\right) .{ }^{13} \mathrm{C}$ NMR (62.9 MHz, $\left.d_{6}-\mathrm{DMSO}\right): \delta 168.8$ (NHC(O)), $130.1\left(\mathrm{Ar}-\mathrm{C}_{\text {ipso }}\right), 124.8\left(\mathrm{Ar}-\mathrm{C}_{\text {meta }}\right), 127.7\left(\mathrm{Ar}-\mathrm{C}_{\text {ortho }}\right), 53.3\left(\mathrm{CH}_{2} \mathrm{~N}_{\left(\mathrm{CH}_{3}\right)}\right), 43.0\left(\mathrm{CH}_{3}\right), 31.7$ $\left(\mathrm{CH}_{2} \mathrm{C}(\mathrm{O})\right)$.

$\boldsymbol{N}$-(2-Methoxy-acetyl)- $\boldsymbol{N}^{\prime}$-(acetyl)-1,2-diamidobenzene (1i). Brownish solid. Yield: $90 \% .{ }^{1} \mathrm{H}$ NMR (250 MHz, $d_{6}$-DMSO): $\delta 9.81$ (brs, $1 \mathrm{H}, \mathrm{NHC}(\mathrm{O})$ ), 9.27 (brs, 1H, NHC(O)), 7.89 (d, 1H, Ar-H), 7.48 (d, $1 \mathrm{H}, \mathrm{Ar}-\mathrm{H}), 2.27(\mathrm{~m}, 2 \mathrm{H}, \mathrm{Ar}-\mathrm{H}), 4.12\left(\mathrm{~s}, 2 \mathrm{H}, \mathrm{CH}_{2} \mathrm{O}\right), 3.48\left(\mathrm{~s}, 3 \mathrm{H}, \mathrm{CH}_{3} \mathrm{O}\right), 2.11\left(\mathrm{~s}, 3 \mathrm{H}, \mathrm{CH}_{3}\right) .{ }^{13} \mathrm{C}$ NMR (62.9 MHz, $d_{6}$-DMSO): $\delta 169.1$ and $167.9(\mathrm{NHC}(\mathrm{O})), 131.6,131.3,130.5,126.9,126.2$, and 125.6 $(\mathrm{Ar}-\mathrm{C}), 72.5\left(\mathrm{CH}_{2} \mathrm{O}\right), 59.6\left(\mathrm{OCH}_{3}\right), 22.7\left(\mathrm{CH}_{3}\right)$.

$N$-(2-Chloro-acetyl)- $N^{\prime}$-(acetyl)-1,2-diamidobenzene (1j). Light red solid. Yield: $86 \%$. ${ }^{1} \mathrm{H}$ NMR (400 $\mathrm{MHz}, d_{6}$-DMSO): $\delta 9.55(\mathrm{~s}, 1 \mathrm{H}, \mathrm{NHC}(\mathrm{O})), 9.53(\mathrm{~s}, 1 \mathrm{H}, \mathrm{NHC}(\mathrm{O})), 7.57(\mathrm{~m}, 1 \mathrm{H}, \mathrm{Ar}-\mathrm{H}), 7.47(\mathrm{~m}, 1 \mathrm{H}$, $\mathrm{Ar}-\mathrm{H}), 7.17(\mathrm{~m}, 2 \mathrm{H}, \mathrm{Ar}-\mathrm{H}), 4.33\left(\mathrm{~s}, 2 \mathrm{H}, \mathrm{CH}_{2}\right), 2.08\left(\mathrm{~s}, 3 \mathrm{H}, \mathrm{CH}_{3}\right) .{ }^{13} \mathrm{C} \mathrm{NMR}\left(100 \mathrm{MHz}, d_{6}-\mathrm{DMSO}\right): \delta$ 169.and 165.0 (NHC(O)), 130.8, 130.0, 125.4, 125.0, 124.9, and 124.8 (Ar-C), $43.4\left(\mathrm{CH}_{2}\right), 23.7\left(\mathrm{CH}_{3}\right)$.

$\boldsymbol{N}$-(3-Chloro-propionyl)- $\boldsymbol{N}^{\prime}$-(acetyl)-1,2-diamidobenzene (1k). Light red solid. Yield: 92\%. ${ }^{1} \mathrm{H}$ NMR (400 MHz, $\left.d_{6}-\mathrm{DMSO}\right): \delta 9.51(\mathrm{~s}, 1 \mathrm{H}, \mathrm{NHC}(\mathrm{O})), 9.23(\mathrm{~s}, 1 \mathrm{H}, \mathrm{NHC}(\mathrm{O})), 7.54(\mathrm{~m}, 2 \mathrm{H}, \mathrm{Ar}-\mathrm{H}), 7.13(\mathrm{~m}$, $2 \mathrm{H}, \mathrm{Ar}-\mathrm{H}), 3.89\left(\mathrm{t}, 2 \mathrm{H},\left(\mathrm{CH}_{2} \mathrm{C}(\mathrm{O})\right), 2.86\left(\mathrm{t}, 2 \mathrm{H}, \mathrm{CH}_{2} \mathrm{Cl}\right), 2.06\left(\mathrm{~s}, 3 \mathrm{H}, \mathrm{CH}_{3}\right) .{ }^{13} \mathrm{C} \mathrm{NMR}\left(100 \mathrm{MHz}, d_{6^{-}}\right.\right.$ DMSO): $\delta 168.6$ and $168.4(\mathrm{NHC}(\mathrm{O})), 130.7,130.1,125.0,124.8,124.7$, and $124.5(\mathrm{Ar}-\mathrm{C}), 40.9$ $\left(\mathrm{CH}_{2} \mathrm{C}(\mathrm{O})\right), 39.0\left(\mathrm{CH}_{2} \mathrm{Cl}\right), 23.7\left(\mathrm{CH}_{3}\right)$. 
$N$-(3-Chloro-propionyl)- $N^{\prime}$-(2-Chloro-acetyl)-1,2-diamidobenzene (11). Light red solid. Yield: 85\%.

${ }^{1} \mathrm{H}$ NMR $\left(250 \mathrm{MHz}, d_{6}\right.$-DMSO): $\delta 9.75(\mathrm{~s}, 1 \mathrm{H}, \mathrm{NHC}(\mathrm{O})), 9.51(\mathrm{~s}, 1 \mathrm{H}, \mathrm{NHC}(\mathrm{O})), 7.52(\mathrm{~m}, 2 \mathrm{H}, \mathrm{Ar}-\mathrm{H})$, $7.20(\mathrm{~m}, 2 \mathrm{H}, \mathrm{Ar}-\mathrm{H}), 4.31\left(\mathrm{~s}, 2 \mathrm{H}, \mathrm{C}(\mathrm{O}) \mathrm{CH}_{2} \mathrm{Cl}\right), 3.83\left(\mathrm{t}, 2 \mathrm{H}, \mathrm{CH}_{2} \mathrm{C}(\mathrm{O})\right), 2.80\left(\mathrm{t}, 2 \mathrm{H}, \mathrm{CH}_{2} \mathrm{Cl}\right) .{ }^{13} \mathrm{C} \mathrm{NMR}$ (62.9 MHz, $d_{6}$-DMSO): $\delta 168.7$ and $165.0(\mathrm{NHC}(\mathrm{O})), 130.6,130.0,125.3,125.2,124.9$, and 113.4 $(\mathrm{Ar}-\mathrm{C}), 43.4\left(\mathrm{C}(\mathrm{O}) \mathrm{CH}_{2} \mathrm{Cl}\right), 40.8\left(\mathrm{CH}_{2} \mathrm{C}(\mathrm{O})\right), 38.9\left(\mathrm{CH}_{2} \mathrm{Cl}\right)$.

$\boldsymbol{N}$-(2-Dimethylamino-acetyl)- $\boldsymbol{N}^{\prime}$-(acetyl)-1,2-diamidobenzene (1m). Brownish oil. Yield: $92 \%$. ${ }^{1} \mathrm{H}$ NMR (400 MHz, $d_{6}$-DMSO): $\delta 10.01(\mathrm{~s}, 1 \mathrm{H}, \mathrm{NHC}(\mathrm{O})), 9.88(\mathrm{~s}, 1 \mathrm{H}, \mathrm{NHC}(\mathrm{O})), 7.73(\mathrm{~d}, 1 \mathrm{H}, \mathrm{Ar}-\mathrm{H}), 7.39$ (d, 1H, Ar-H), $7.14(\mathrm{~m}, 2 \mathrm{H}, \mathrm{Ar}-\mathrm{H}), 3.40\left(\mathrm{~s}, 2 \mathrm{H}, \mathrm{CH}_{2} \mathrm{~N}\left(\mathrm{CH}_{3}\right)_{2}\right), 2.50\left(\mathrm{~s}, 6 \mathrm{H}, \mathrm{N}\left(\mathrm{CH}_{3}\right)_{2}\right), 2.09(\mathrm{~s}, 3 \mathrm{H}$, $\left.\mathrm{CH}_{3}\right) .{ }^{13} \mathrm{C}$ NMR (100 MHz, $d_{6}$-DMSO): $\delta 168.9$ and $166.8(\mathrm{NHC}(\mathrm{O})), 130.9,129.9,125.4,125.3,124.7$, and $124.0(\mathrm{Ar}-\mathrm{C}), 61.4\left(\mathrm{CH}_{2} \mathrm{~N}\left(\mathrm{CH}_{3}\right)_{2}, 44.7\left(\mathrm{~N}\left(\mathrm{CH}_{3}\right)_{2}\right), 23.1\left(\mathrm{CH}_{3}\right)\right.$.

$N$-(3-Dimethylamino-propionyl)- $N^{\prime}$-(acetyl)-1,2-diamidobenzene (1n). Brown oil. Yield: $86 \%$. ${ }^{1} \mathrm{H}$ NMR (400 MHz, $d_{6}$-DMSO): $\delta 10.10(\mathrm{~s}, 1 \mathrm{H}, \mathrm{NHC}(\mathrm{O})$ ), 9.83 (s, 1H, NHC(O)), 7.59 (m, 2H, Ar-H); $7.09(\mathrm{~m}, 2 \mathrm{H}, \mathrm{Ar}-\mathrm{H}), 3.16\left(\mathrm{t}, 2 \mathrm{H}, \mathrm{CH}_{2} \mathrm{C}(\mathrm{O})\right), 2.81\left(\mathrm{t}, 2 \mathrm{H}, \mathrm{CH}_{2} \mathrm{~N}\left(\mathrm{CH}_{3}\right)_{2}\right), 2.63\left(\mathrm{~s}, 6 \mathrm{H}, \mathrm{N}\left(\mathrm{CH}_{3}\right)_{2}\right), 2.12$ (s, $\left.3 \mathrm{H}, \mathrm{CH}_{3}\right) .{ }^{13} \mathrm{C}-\mathrm{NMR}\left(100 \mathrm{MHz}, d_{6}\right.$-DMSO,): $\delta 168.6$ and $168.5(\mathrm{NHC}(\mathrm{O})), 130.5,129.8,124.7,124.6$, 124.4, and $124.3(\mathrm{Ar}-\mathrm{C}), 53.1\left(\mathrm{CH}_{2} \mathrm{~N}\left(\mathrm{CH}_{3}\right)_{2}\right), 42.7\left(\mathrm{~N}\left(\mathrm{CH}_{3}\right)_{2}\right), 31.4\left(\mathrm{CH}_{2} \mathrm{C}(\mathrm{O})\right), 23.7\left(\mathrm{CH}_{3}\right)$.

$N$-(3-Dimethylamino-propionyl)- $N^{\prime}$-(2-dimethylamino-acetyl)-1,2-diamidobenzene (10). Brown oil. Yield: 85\%. ${ }^{1} \mathrm{H}$ NMR (250 MHz, $d_{6}$-DMSO): $\delta 10.39$ (brs, 1H, NHC(O)), 9.58 (brs, 1H, NHC(O)), 7.63 $(\mathrm{d}, 1 \mathrm{H}, \mathrm{Ar}-\mathrm{H}), 7.40(\mathrm{~d}, 1 \mathrm{H}, \mathrm{Ar}-\mathrm{H}), 7.09(\mathrm{~m}, 2 \mathrm{H}, \mathrm{Ar}-\mathrm{H}), 3.31\left(\mathrm{~s}, 2 \mathrm{H}, \mathrm{CH}_{2} \mathrm{C}(\mathrm{O})\right), 3.25\left(\mathrm{t}, 2 \mathrm{H}, C \mathrm{H}_{2} \mathrm{C}(\mathrm{O})\right)$, $2.84\left(\mathrm{t}, 2 \mathrm{H}, \mathrm{CH}_{2} \mathrm{~N}, 2.50\left(\mathrm{~s}, 6 \mathrm{H}, \mathrm{N}\left(\mathrm{CH}_{3}\right)_{2}\right), 2.44\left(\mathrm{~s}, 6 \mathrm{H}, \mathrm{N}\left(\mathrm{CH}_{3}\right)_{2}\right) .{ }^{13} \mathrm{C}-\mathrm{NMR}\left(62.9 \mathrm{MHz}, d_{6}\right.\right.$-DMSO): $\delta$ 168.9 and $167.7(\mathrm{NHC}(\mathrm{O})), 131.4$ and $129.2\left(\mathrm{Ar}-\mathrm{C}_{i p s o}\right), 125.7$ and $125.6\left(\mathrm{Ar}-\mathrm{C}_{\text {meta }}\right), 124.8$ and 123.9 (Ar- $\left.\mathrm{C}_{\text {ortho }}\right), 62.1\left(\mathrm{CH}_{2} \mathrm{C}(\mathrm{O})\right), 52.9\left(\mathrm{CH}_{2} \mathrm{~N}\right), 45.1$ and $42.6\left(\mathrm{~N}\left(\mathrm{CH}_{3}\right)_{2}\right), 34.0\left(\mathrm{CH}_{2} \mathrm{C}(\mathrm{O})\right)$. 
$\boldsymbol{N}, \boldsymbol{N}^{\prime}$-Dimethyl-1,2-diaminobenzene (2a). Colourless solid, mp: $27{ }^{\circ} \mathrm{C}$. Yield $86 \% .{ }^{1} \mathrm{H}$ NMR $(250$ $\left.\mathrm{MHz}, \mathrm{C}_{6} \mathrm{D}_{6}\right): \delta 7.0(\mathrm{~m}, 2 \mathrm{H}, \mathrm{Ar}-\mathrm{H}), 6.8(\mathrm{~m}, 2 \mathrm{H}, \mathrm{Ar}-\mathrm{H}), 3.2(\mathrm{~s}, 2 \mathrm{H}, \mathrm{NH}), 2.9\left(\mathrm{~s}, 6 \mathrm{H}, \mathrm{CH}_{3}\right) .{ }^{13} \mathrm{C} \mathrm{NMR}$ (62.9 MHz, $\left.\mathrm{C}_{6} \mathrm{D}_{6}\right): \delta 138.2\left(\mathrm{Ar}-\mathrm{C}_{\text {ipso }}\right), 118.9\left(\mathrm{Ar}-\mathrm{C}_{\text {meta }}\right), 110.3\left(\mathrm{Ar}-\mathrm{C}_{\text {ortho }}\right), 30.9\left(\mathrm{CH}_{3}\right)$.

$\boldsymbol{N}, \boldsymbol{N}^{\prime}$-Diethyl-1,2-diaminobenzene (2b). Colourless oil. Yield 80\%. ${ }^{1} \mathrm{H}$ NMR $\left(200 \mathrm{MHz}, \mathrm{CDCl}_{3}\right): \delta$ $7.30(\mathrm{~m}, 2 \mathrm{H}, \mathrm{Ar}-\mathrm{H}), 7.10(\mathrm{~m}, 2 \mathrm{H}, \mathrm{Ar}-\mathrm{H}), 3.61(\mathrm{~s}, 2 \mathrm{H}, \mathrm{NH}), 3.47\left(\mathrm{q}, 4 \mathrm{H}, \mathrm{NCH}_{2}\right), 1.68\left(\mathrm{t}, 6 \mathrm{H}, \mathrm{CH}_{3}\right) .{ }^{13} \mathrm{C}$ $\operatorname{NMR}\left(50.3 \mathrm{MHz}, \mathrm{CDCl}_{3}\right): \delta 136.9\left(\mathrm{Ar}-\mathrm{C}_{\text {ipso }}\right), 118.5\left(\mathrm{Ar}-\mathrm{C}_{\text {meta }}\right), 110.8\left(\mathrm{Ar}-\mathrm{C}_{\text {ortho }}\right), 38.3\left(\mathrm{NCH}_{2}\right), 14.5$ $\left(\mathrm{CH}_{3}\right)$.

$\boldsymbol{N}, \boldsymbol{N}^{\prime}$-Bis(2-methoxy-ethyl)-1,2-diaminobenzol (2c). Brownish oil. Yield $80 \% .{ }^{1} \mathrm{H}$ NMR $(250 \mathrm{MHz}$, $\left.\mathrm{C}_{6} \mathrm{D}_{6}\right): \delta 6.95(\mathrm{~m}, 2 \mathrm{H}, \mathrm{Ar}-\mathrm{H}), 6.60(\mathrm{~m}, 2 \mathrm{H}, \mathrm{Ar}-\mathrm{H}), 3.80(\mathrm{~s}, 2 \mathrm{H}, \mathrm{NH}), 3.30\left(\mathrm{t}, 4 \mathrm{H}, \mathrm{OCH}_{2}\right), 3.00(\mathrm{t}, 4 \mathrm{H}$, $\left.\mathrm{NCH}_{2}\right), 2.95\left(\mathrm{~s}, 6 \mathrm{H}, \mathrm{CH}_{3}\right) .{ }^{13} \mathrm{C} \mathrm{NMR}\left(62.9 \mathrm{MHz}, \mathrm{C}_{6} \mathrm{D}_{6}\right): \delta 137.0\left(\mathrm{Ar}-\mathrm{C}_{i p s o}\right), 119.6\left(\mathrm{Ar}-\mathrm{C}_{\text {meta }}\right), 112.4$ $\left(\mathrm{Ar}-\mathrm{C}_{\text {ortho }}\right), 71.2\left(\mathrm{OCH}_{2}\right), 58.3\left(\mathrm{NCH}_{2}\right), 44.1\left(\mathrm{OCH}_{3}\right)$.

$\boldsymbol{N}, \boldsymbol{N}^{\prime}$-Bis(3-methoxy-propyl)-1,2-diaminobenzol (2d). Brownish oil. Yield 77\%. ${ }^{1} \mathrm{H}$ NMR $(250 \mathrm{MHz}$, $d_{8}$-THF): $\delta 6.53(\mathrm{~m}, 4 \mathrm{H}, \mathrm{Ar}-\mathrm{H}), 3.85(\mathrm{brs}, 2 \mathrm{H}, \mathrm{NH}), 3.48\left(\mathrm{t}, 4 \mathrm{H}, \mathrm{CH}_{2} \mathrm{O}\right), 3.24\left(\mathrm{~s}, 6 \mathrm{H}, \mathrm{CH}_{3}\right), 3.18(\mathrm{t}, 4 \mathrm{H}$, $\left.\mathrm{NCH}_{2}\right), 1.87\left(\mathrm{dt}, 4 \mathrm{H}, \mathrm{CH}_{2} \mathrm{CH}_{2} \mathrm{CH}_{2}\right) .{ }^{13} \mathrm{C} \mathrm{NMR}\left(62.9 \mathrm{MHz}, d_{8}-\mathrm{THF}\right): \delta 138.3\left(\mathrm{Ar}-\mathrm{C}_{i p s o}\right), 118.9$ $\left(\mathrm{Ar}-\mathrm{C}_{\text {meta }}\right), 111.3\left(\mathrm{Ar}-\mathrm{C}_{\text {ortho }}\right), 71.9\left(\mathrm{CH}_{2} \mathrm{O}\right), 58.2\left(\mathrm{NCH}_{2}\right), 42.7\left(\mathrm{CH}_{3}\right), 30.5\left(\mathrm{CH}_{2} \mathrm{CH}_{2} \mathrm{CH}_{2}\right)$.

$\boldsymbol{N}, \boldsymbol{N}^{\prime}$-Bis(2-dimethylamino-ethyl)-1,2-diaminobenzene (2g). Yellow oil. Yield 84\%. ${ }^{1} \mathrm{H}$ NMR (250 $\left.\mathrm{MHz}, \mathrm{C}_{6} \mathrm{D}_{6}\right): \delta 6.98(\mathrm{~m}, 2 \mathrm{H}, \mathrm{Ar}-\mathrm{H}), 6.76(\mathrm{~m}, 2 \mathrm{H}, \mathrm{Ar}-\mathrm{H}), 4.20$ (brs, 2H, NH), 2.96 (t, 4H, HNCH $\left.H_{2}\right), 2.38$ $\left(\mathrm{t}, 4 \mathrm{H}, \mathrm{CH}_{2} \mathrm{~N}\left(\mathrm{CH}_{3}\right)_{2}\right), 1.97\left(\mathrm{~s}, 12 \mathrm{H}, \mathrm{CH}_{3}\right) .{ }^{13} \mathrm{C} \mathrm{NMR}\left(62.9 \mathrm{MHz}, \mathrm{C}_{6} \mathrm{D}_{6}\right): \delta 138.2\left(\mathrm{Ar}-\mathrm{C}_{\text {ipso }}\right), 119.3$ $\left(\mathrm{Ar}-\mathrm{C}_{\text {meta }}\right), 111.6\left(\mathrm{Ar}-\mathrm{C}_{\text {ortho }}\right), 58.2\left(\mathrm{NCH}_{2}\right), 45.1\left(\mathrm{CH}_{3}\right), 41.9\left(\mathrm{CH}_{2} \mathrm{~N}\left(\mathrm{CH}_{3}\right)_{2}\right)$. 
$\boldsymbol{N}, \boldsymbol{N}^{\prime}$-Bis(3-dimethylamino-propyl)-1,2-diaminobenzene (2h). Yellow oil. Yield 82\%. ${ }^{1} \mathrm{H}$ NMR (250 $\left.\mathrm{MHz}, \mathrm{C}_{6} \mathrm{D}_{6}\right): \delta 6.95(\mathrm{~m}, 2 \mathrm{H}, \mathrm{Ar}-\mathrm{H}), 6.60(\mathrm{~m}, 2 \mathrm{H}, \mathrm{Ar}-\mathrm{H}), 4.1(\mathrm{brs}, 2 \mathrm{H}, \mathrm{NH}), 2.95\left(\mathrm{t}, 4 \mathrm{H}, \mathrm{NCH}_{2}\right), 2.20(\mathrm{t}$, $\left.4 \mathrm{H}, \mathrm{CH}_{2} \mathrm{~N}\left(\mathrm{CH}_{3}\right)_{2}\right), 2.05\left(\mathrm{~s}, 12 \mathrm{H}, \mathrm{CH}_{3}\right), 1.80\left(\mathrm{dt}, \mathrm{CH}_{2} \mathrm{CH}_{2} \mathrm{CH}_{2}\right) .{ }^{13} \mathrm{C} \mathrm{NMR}\left(62.9 \mathrm{MHz}, \mathrm{C}_{6} \mathrm{D}_{6}\right): \delta 138.1$

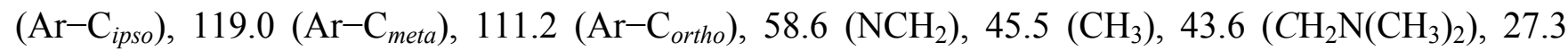
$\left(\mathrm{CH}_{2} \mathrm{CH}_{2} \mathrm{CH}_{2}\right)$.

$\boldsymbol{N}$-(2-Methoxy-ethyl)- $\boldsymbol{N}^{\prime}$-(ethyl)-1,2-diaminobenzene (2i). Brownish oil. Yield 75\%. ${ }^{1} \mathrm{H}$ NMR (250 $\left.\mathrm{MHz}, \mathrm{C}_{6} \mathrm{D}_{6}\right): \delta 6.98(\mathrm{~m}, 2 \mathrm{H}, \mathrm{Ar}-\mathrm{H}), 6.60(\mathrm{~m}, 2 \mathrm{H}, \mathrm{Ar}-\mathrm{H}), 3.71$ (brs, $\left.1 \mathrm{H}, \mathrm{NH}\right), 3.40\left(\mathrm{t}, 2 \mathrm{H}, \mathrm{CH}_{2} \mathrm{O}\right), 3.21$ (brs, 1H, NH), 3.16 (t, 2H, $\mathrm{NCH}_{2} \mathrm{CH}_{2}$ ), 3.06 (s, 3H, $\left.\mathrm{OCH}_{3}\right), 2.76$ (q, 2H, $\left.\mathrm{NCH}_{2} \mathrm{CH}_{3}\right), 0.88\left(\mathrm{t}, 3 \mathrm{H}, \mathrm{CH}_{3}\right)$. ${ }^{13} \mathrm{C}$ NMR (62.9 MHz, $\left.\mathrm{C}_{6} \mathrm{D}_{6}\right): \delta 138.4$ and $137.4\left(\mathrm{Ar}-\mathrm{C}_{i p s o}\right), 122.5$ and $122.0\left(\mathrm{Ar}-\mathrm{C}_{\text {meta }}\right), 112.4$ and 111.7 (Ar- $\left.\mathrm{C}_{\text {ortho }}\right), 74.3\left(\mathrm{CH}_{2} \mathrm{O}\right), 58.3\left(\mathrm{NCH}_{2} \mathrm{CH}_{2}\right), 44.1\left(\mathrm{OCH}_{3}\right), 38.7\left(\mathrm{NCH}_{2} \mathrm{CH}_{3}\right), 14.8\left(\mathrm{CH}_{3}\right)$.

$\boldsymbol{N}$-(2-Dimethylamino-ethyl)- $\boldsymbol{N}^{\prime}$-(ethyl)-1,2-diaminobenzene (2m). Yellow oil. Yield 77\%. ${ }^{1} \mathrm{H}$ NMR $\left(250 \mathrm{MHz}, \mathrm{C}_{6} \mathrm{D}_{6}\right): \delta 6.87(\mathrm{~m}, 2 \mathrm{H}, \mathrm{Ar}-\mathrm{H}), 6.60(\mathrm{~m}, 2 \mathrm{H}, \mathrm{Ar}-\mathrm{H}), 4.0$ (brs, $\left.1 \mathrm{H}, \mathrm{NH}\right), 3.43$ (brs, 1H, NH), 2.81 (t, 2H, $\left.\mathrm{NCH}_{2} \mathrm{CH}_{2}\right), 2.76\left(\mathrm{q}, 2 \mathrm{H}, \mathrm{NCH}_{2} \mathrm{CH}_{3}\right), 2.29\left(\mathrm{t}, 2 \mathrm{H}, \mathrm{CH}_{2} \mathrm{~N}\left(\mathrm{CH}_{3}\right)_{2}\right), 1.92\left(\mathrm{~s}, 6 \mathrm{H}, \mathrm{N}\left(\mathrm{CH}_{3}\right)_{2}\right), 0.93$ $\left(\mathrm{t}, 3 \mathrm{H}, \mathrm{CH}_{3}\right) .{ }^{13} \mathrm{C} \mathrm{NMR}\left(62.9 \mathrm{MHz}, \mathrm{C}_{6} \mathrm{D}_{6}\right): \delta 138.1$ and $137.9\left(\mathrm{Ar}-\mathrm{C}_{i p s o}\right), 119.4$ and $119.1\left(\mathrm{Ar}-\mathrm{C}_{\text {meta }}\right)$, 111.8 and $111.4\left(\mathrm{Ar}-\mathrm{C}_{\text {ortho }}\right), 58.4\left(\mathrm{NCH}_{2} \mathrm{CH}_{2}\right), 45.1\left(\mathrm{~N}\left(\mathrm{CH}_{3}\right)_{2}\right), 41.9\left(\mathrm{CH}_{2} \mathrm{~N}\left(\mathrm{CH}_{3}\right)_{2}\right), 38.8\left(\mathrm{NCH}_{2} \mathrm{CH}_{3}\right)$, $14.8\left(\mathrm{CH}_{3}\right)$.

$N$-(3-Dimethylamino-propyl)- $N^{\prime}$-(ethyl)-1,2-diaminobenzene (2n). Yellow oil. Yield 80\%. ${ }^{1} \mathrm{H}$ NMR $\left(400 \mathrm{MHz}, \mathrm{C}_{6} \mathrm{D}_{6}\right): \delta 6.98(\mathrm{~m}, 2 \mathrm{H}, \mathrm{Ar}-\mathrm{H}), 6.71(\mathrm{~m}, 2 \mathrm{H}, \mathrm{Ar}-\mathrm{H}), 3.81$ (brs, 2H, NH), $3.01(\mathrm{t}, 2 \mathrm{H}$, $\left.\mathrm{NCH}_{2} \mathrm{CH}_{2}\right), 2.85$ (q, 2H, $\left.\mathrm{NCH}_{2} \mathrm{CH}_{3}\right), 2.11\left(\mathrm{t}, 2 \mathrm{H}, \mathrm{CH}_{2} \mathrm{~N}\left(\mathrm{CH}_{3}\right)_{2}\right), 2.04\left(\mathrm{~s}, 6 \mathrm{H}, \mathrm{N}\left(\mathrm{CH}_{3}\right)_{2}\right), 1.52(\mathrm{dt}, 2 \mathrm{H}$, $\left.\mathrm{CH}_{2} \mathrm{CH}_{2} \mathrm{CH}_{2}\right), 0.98\left(\mathrm{t}, 3 \mathrm{H}, \mathrm{CH}_{3}\right) .{ }^{13} \mathrm{C} \mathrm{NMR}\left(100 \mathrm{MHz}, \mathrm{C}_{6} \mathrm{D}_{6}\right): \delta 138.2$ and $137.9\left(\mathrm{Ar}-\mathrm{C}_{i p s o}\right), 119.4$ and

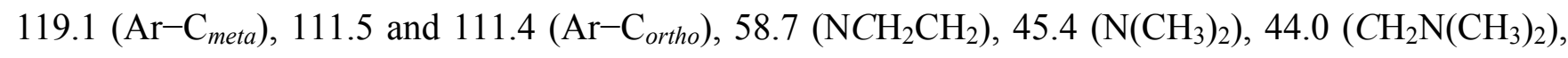
$38.8\left(\mathrm{NCH}_{2} \mathrm{CH}_{3}\right), 26.9\left(\mathrm{CH}_{2} \mathrm{CH}_{2} \mathrm{CH}_{2}\right), 15.0\left(\mathrm{CH}_{3}\right)$ 
$N$-(3-Dimethylamino-propyl)- $N^{\prime}$-(2-dimethylamino-ethyl)-1,2-diaminobenzene (2o). Orange oil. Yield 75\%. ${ }^{1} \mathrm{H}-\mathrm{NMR}\left(250 \mathrm{MHz}, \mathrm{C}_{6} \mathrm{D}_{6}\right): \delta 6.97(\mathrm{~m}, 2 \mathrm{H}, \mathrm{Ar}-\mathrm{H}), 6.58(\mathrm{~m}, 2 \mathrm{H}, \mathrm{Ar}-\mathrm{H}), 4.55$ (brs, 1H, NH), 4.14 (brs, $1 \mathrm{H}, \mathrm{NH}), 3.05$ (t, 2H, $\left.\mathrm{HNCH}_{2}\right), 2.96$ (t, 2H, $\left.\mathrm{HNCH}_{2}\right), 2.34\left(\mathrm{t}, 2 \mathrm{H}, \mathrm{CH}_{2} \mathrm{~N}_{(}\left(\mathrm{CH}_{3}\right)_{2}\right), 2.12(\mathrm{t}, 2 \mathrm{H}$, $\left.\mathrm{CH}_{2} \mathrm{~N}\left(\mathrm{CH}_{3}\right)_{2}\right), 2.06\left(\mathrm{~s}, 6 \mathrm{H}, \mathrm{N}\left(\mathrm{CH}_{3}\right)_{2}\right), 1.87\left(\mathrm{~s}, 6 \mathrm{H}, \mathrm{N}\left(\mathrm{CH}_{3}\right)_{2}\right), 1.52\left(\mathrm{dt}, 2 \mathrm{H}, \mathrm{CH}_{2} \mathrm{CH}_{2} \mathrm{CH}_{2}\right) .{ }^{13} \mathrm{C} \mathrm{NMR}$ $\left(62.9 \mathrm{MHz}, \mathrm{C}_{6} \mathrm{D}_{6}\right): \delta 138.4$ and $137.9\left(\mathrm{Ar}-\mathrm{C}_{i p s o}\right), 119.2$ and $118.9\left(\mathrm{Ar}-\mathrm{C}_{m e t a}\right), 111.2$ and 110.9 $\left(\mathrm{Ar}-\mathrm{C}_{\text {ortho }}\right), 59.0\left(\mathrm{HNCH}_{2}\right), 58.4\left(\mathrm{HNCH}_{2}\right), 45.4\left(\mathrm{~N}\left(\mathrm{CH}_{3}\right)_{2}\right), 45.1\left(\mathrm{~N}\left(\mathrm{CH}_{3}\right)_{2}\right), 44.2\left(\mathrm{CH}_{2} \mathrm{~N}_{(}\left(\mathrm{CH}_{3}\right)_{2}\right), 41.7$ $\left(\mathrm{CH}_{2} \mathrm{~N}\left(\mathrm{CH}_{3}\right)_{2}\right), 26.7\left(\mathrm{CH}_{2} \mathrm{CH}_{2} \mathrm{CH}_{2}\right)$. 